\title{
Improvement of Type 2 Diabetes Mellitus in Obese and Non-Obese Patients after the Duodenal Switch Operation
}

\author{
M. Frenken, ${ }^{1}$ E. Y. Cho, ${ }^{1}$ W. K. Karcz, ${ }^{2}$ J. Grueneberger, ${ }^{2}$ and S. Kuesters ${ }^{2}$ \\ ${ }^{1}$ Department of Surgery, St. Josef Hospital Monheim, 40789 Monheim am Rhein, Germany \\ ${ }^{2}$ Department of General and Visceral Surgery, University of Freiburg, Hugstetter Street 55, 79106 Freiburg, Germany
}

Correspondence should be addressed to S. Kuesters, simon.kuesters@uniklinik-freiburg.de

Received 31 August 2010; Accepted 13 January 2011

Academic Editor: Francesco Saverio Papadia

Copyright (C) 2011 M. Frenken et al. This is an open access article distributed under the Creative Commons Attribution License, which permits unrestricted use, distribution, and reproduction in any medium, provided the original work is properly cited.

\begin{abstract}
Introduction. Type 2 diabetes mellitus (T2DM) is one of the most important obesity-related comorbidities. This study was undertaken to characterise the effect of the biliopancreatic diversion with duodenal switch (BPD-DS) in morbidly obese and nonmorbidly obese diabetic patients. Methods. Outcome of 74 obese diabetic patients after BPD-DS and 16 non-obese diabetic patients after BPD or gastric bypass surgery was evaluated. Insulin usage, $\mathrm{HbA}_{1 c}$-levels, and index of HOMA-IR (homeostasis model assessment of insulin resistence) were measured. Results. A substantial fraction of patients is free of insulin and shows an improved insulin sensitivity early after the operation, another fraction gets free of insulin in a 12-month period after the operation and a small fraction of long-term insulin users will not get free of insulin but nevertheless shows an improved metabolic status (less insulin needed, normal $\mathrm{HbA}_{1 \mathrm{c}}$-levels). Conclusion. BPD-DS leads to an improvement of T2DM in obese and non-obese patients. Nevertheless, more data is needed to clarify indications and mechanisms of action and to adjust our operation techniques to the needs of non-obese diabetic patients.
\end{abstract}

\section{Introduction}

Obese patients' lifetime and life quality are not only limited by obesity itself but also by associated disorders, in particular type 2 diabetes mellitus (T2DM). Patients need medication, regular subcutaneous injections, and should keep a diet. Frequently, the diabetes is poorly controlled, which leads to various complications, even failure of organs. Serious vascular diseases may occur, and some patients need dialysis or surgery because of diabetes related complications. Fortunately bariatric surgery shows effects not only on body weight but also on glucose homoeostasis. In the long-term some operations show better results concerning diabetes than concerning weight loss. Patients remain overweight but experience remission from diabetes [1]. The question arises if we should rather talk of "diabetes surgery" than "bariatric surgery" when operating an obese patient with T2DM [2]. The next step would consequently be the "antidiabetic" operation of non-obese patients with inadequately controlled diabetes [3-5]. The "Standards of Medical Care in Diabetes" published yearly by the American Diabetes Association, for the first time, mentions surgical therapy in 2009 [6]. They recommend bariatric surgery for adults with BMI $>35 \mathrm{~kg} / \mathrm{m}^{2}$ and type 2 diabetes, especially if the diabetes or associated comorbidities are difficult to control with lifestyle and pharmacologic therapy. However, they state that there is currently insufficient evidence to generally recommend surgery in patients with BMI $<35 \mathrm{~kg} / \mathrm{m}^{2}$ outside of a research protocol. These recommendations were assumed without any change for 2010 [7]. Similar recommendations were made by the Diabetes Surgery Summit Consensus Conference in 2010: surgery should be considered for patients with BMI $>35 \mathrm{~kg} / \mathrm{m}^{2}$ who are inadequately controlled by lifestyle and medical therapy. A surgical approach may also be appropriate as an nonprimary alternative to treat inadequately controlled diabetes in patients with a BMI of $30-35 \mathrm{~kg} / \mathrm{m}^{2}$ [8]. The biliopancreatic diversion with duodenal switch (BPD-DS) is a well-established bariatric operation with encouraging effects on glucose homeostasis and diabetes $[9,10]$. In the present study, we present our first results concerning improvement of T2DM in morbidly obese and also in nonmorbidly obese patients. As outcome parameters usage of 


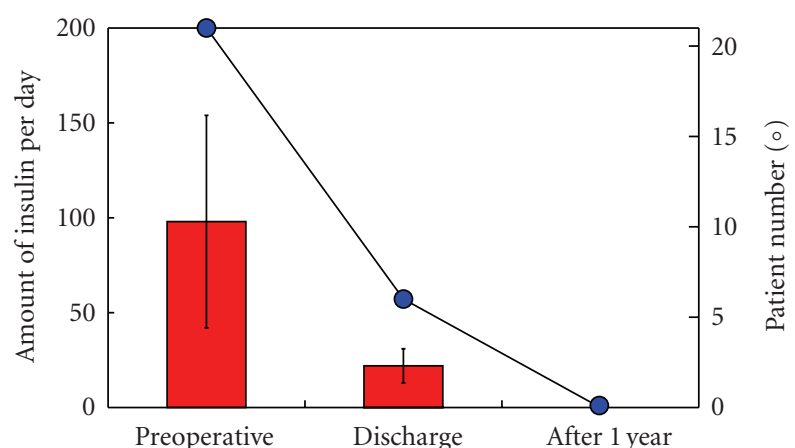

FIGURE 1: Reduction of insulin usage prior to BPD-DS, at discharge and one year after the operation. Blue dots indicate the number of patients in need of insulin (right scale). Red bars indicate the mean amount of insulin used per day (left scale, whiskers indicate standard deviation).

insulin, $\mathrm{HbA}_{1 \mathrm{c}}$-levels and HOMA-IR index were measured [11].

\section{Patients and Methods}

Patients: as a first group, 21 obese patients ( 9 male/ 12 female) with insulin-dependent T2DM received a biliopancreatic diversion with duodenal switch (BPD-DS). Mean age was 51 years (26-67), mean preoperative BMI was $46 \mathrm{~kg} / \mathrm{m}^{2}$ (3556 ), mean duration of diabetes was 10,5 years $(2-30)$, and preoperative duration of insulin treatment was 6.5 years $(0,5-25)$ with a mean usage of $98(25-250)$ units of insulin per day. Mean preoperative $\mathrm{HbA}_{1 \mathrm{c}}$ was 9,5\% $(6,0-14,0)$. Body weight, usage of insulin or oral antidiabetic drugs and $\mathrm{HbA}_{1 \mathrm{c}}$ levels were evaluated 3, 6, and 12 months after surgery.

Retrospective analysis of sixteen diabetic patients $(8$ male $/ 8$ female) with a BMI $<35 \mathrm{~kg} / \mathrm{m}^{2}$ (mean BMI 32, 26$34,5)$ was also performed, mean age was 56 years (3668 ). Mean duration of diabetes was 16 years (4-40), mean duration of insulin therapy was 6 years (1-12), and mean daily amount of insulin used was $92 \mathrm{IU}(30-140)$. These patients received either BPD-DS $(n=7)$, BPD-Scopinaro $(n=5)$, or Roux-en-Y gastric bypass $(n=4)$. All patients with BMI $<35 \mathrm{~kg} / \mathrm{m}^{2}$ knew that intestinal bypass surgery is not yet a standard procedure to treat diabetes and signed an informed consent.

To evaluate early improvements of insulin sensitivity, HOMA-IR index was measured at the day of surgery and 3, 7 , 14, and 21 days after BPD-DS in a group of 27 obese patients (21 patients using insulin before surgery). Mean preoperative HOMA-IR index was 14,3.

Seventy-four patients after BPD-DS operations between February 2005 and January 2009 were retrospectively evaluated concerning postoperative outcome in relation to preoperative duration of insulin therapy. Mean age was 50 years (26-68), mean BMI was $47 \mathrm{~kg} / \mathrm{m}^{2}$. BPD-DS operations were conducted with a common channel of $100 \mathrm{~cm}$ in an open approach. All operations were performed at the Department of Surgery, St. Josef Hospital Monheim, Germany.

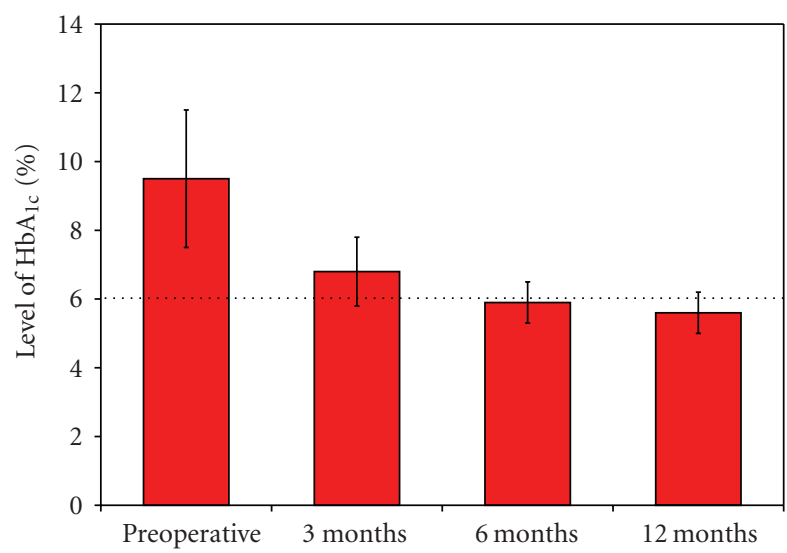

FIgURE 2: $\mathrm{HbA}_{1 \mathrm{c}}$ levels prior to BPD-DS and 3, 6, and 12 months after the operation. Red bars indicate the mean levels of $\mathrm{HbA}_{1 c}$, whiskers indicate standard deviation.

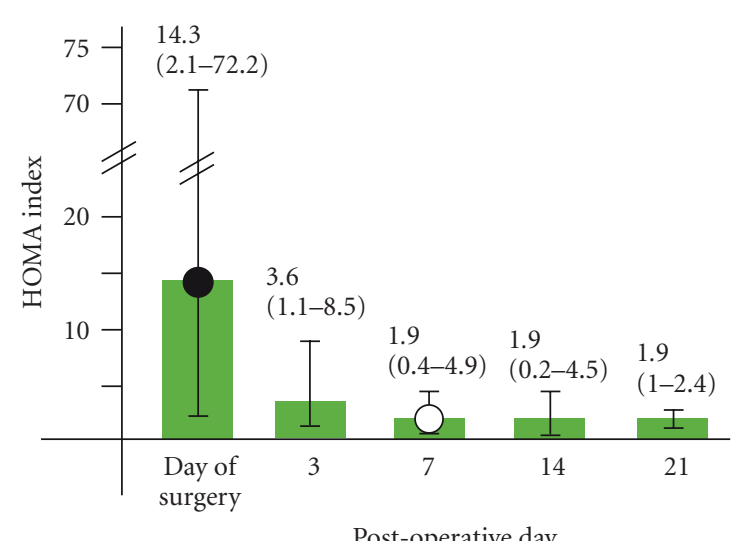

FIGURE 3: HOMA index at the day of surgery and 3, 7, 14, and 21 days after BPD-DS (27 patients). Green bars indicate mean HOMA index (whiskers indicate minimum and maximum values). Seven days after the operation, the mean HOMA index reaches a normal value $<2$.

\section{Results}

3.1. Remission of Insulin-Dependent Type 2 Diabetes Mellitus after $B P D-D S$. In group one, mean preoperative BMI was $46 \mathrm{~kg} / \mathrm{m}^{2}$ (35-56), mean preoperative duration of insulin treatment was 6.5 years with a mean usage of 98 units of insulin per day. At time point of discharge, 15 patients did not need insulin any more and one year after the operation insulin therapy was terminated in all patients (Figure 1). $\mathrm{HbA}_{1 \mathrm{c}}$ levels decreased from preoperatively 9.5 (mean) to 5.9 (mean) and 5.6 (mean) after 6 and 12 months without any dietary restriction (Figure 2). Only one patient needed an antidiabetic drug one year after the operation. In the group of non-obese patients, similar results were seen; at discharge, 13 patients were free of insulin, and, one year after the operation all patients were free of insulin. Patients after BPD-DS and BPD-Scopinaro showed slightly better results than patients after gastric bypass (data not shown). 


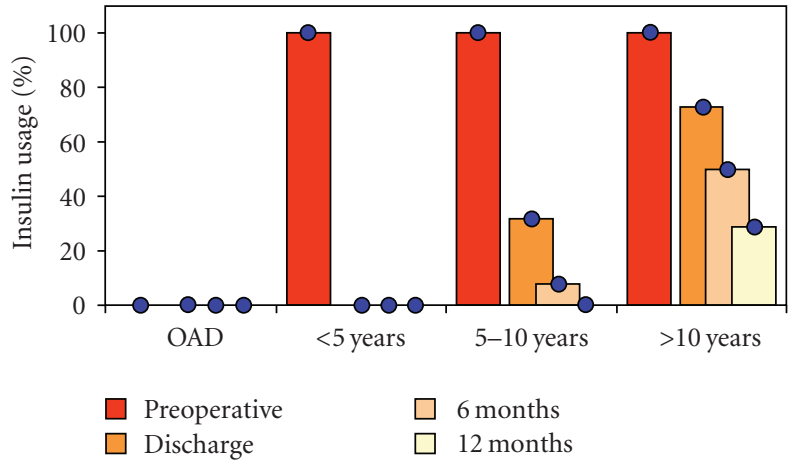

FIGURE 4: Reduction of insulin usage after BPD-DS dependent on preoperative duration of insulin usage. Group I "OAD” was treated by oral antidiabetic drugs only and used no insulin postoperatively. Group II used insulin for less than 5 years preoperatively and needed no insulin at discharge and after. Group III used insulin for 5 to 10 years. Thirty-seven percent of patients in this group needed insulin at discharge (light brown bar), but all patients in this group were free of insulin 1 year after the operation. Group IV used insulin for more than 10 years. Seventy-three percent of patients in this group needed insuline at discharge (light brown bar), 23\% of patients in this group still needed insuline 1 year after the operation (white bar).

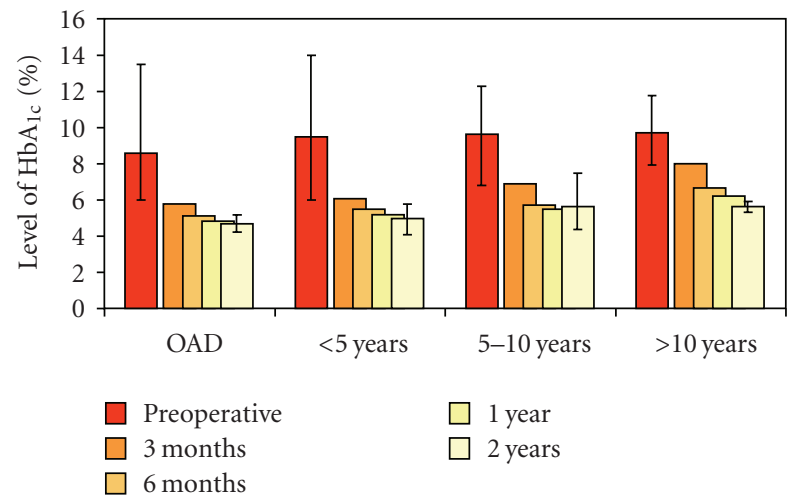

FIGURE 5: $\mathrm{HbA}_{1 \mathrm{c}}$ levels after BPD-DS dependent on preoperative duration of insuline usage. Group I "OAD" was treated by oral antidiabetic drugs only. $\mathrm{HbA}_{1 \mathrm{c}}$ levels were below $6 \%$ as early as 3 months after the operation. Group II used insulin for less than 5 years preoperatively and also showed normal levels of $\mathrm{HbA}_{\mathrm{lc}}$ from 3 months after the operation on. Group III used insulin for 5 to 10 years. Mean $\mathrm{HbA}_{\mathrm{lc}}$ normalized 6 months after the operation. Group IV used insulin for more than 10 years. Mean $\mathrm{HbA}_{1 \mathrm{c}}$ in this group normalized not until 2 years after the operation. Shown are mean values and additionally minimum/maximum values for preoperative and 2 years data.

\subsection{Decrease of Insulin Resistance in the Early Postoperative} Phase. Mean HOMA-IR index decreased from a preoperative value of 14.3 to 3.6 , and 1.9 three and 7 days after surgery (Figure 3). There was no significant difference whether the patients were treated with oral antidiabetics or with insulin. Also, three patients who were discharged from hospital with small amounts of insulin (maximally 36 units per day) had a low postoperative HOMA-IR index of 1.3 to 2.6. No dependence of BMI on the postoperative course of insulin resistance could be detected.

\subsection{Influence of Preoperative Duration of Insulin Therapy on} Postoperative Outcome. According to their need for insulin, patients in group 3 were divided into 4 groups: 15 patients were treated with oral antidiabetic medication, 25 used insulin for less than 5 years, 23 used insulin for 5-10 years, and 11 used insulin for more than 10 years. At discharge from hospital, all patients in groups I and II were free of insulin. Thirty-three percent of patients in group III, still needed insulin at the time of discharge, but all of them were free of insulin 12 months after the operation. In group IV, $73 \%$ needed insulin at the time of discharge, $23 \%$ still needed small amounts of insulin 12 months after the operation (Figure 4). $\mathrm{HbA}_{1 \mathrm{c}}$ levels also decreased continuously in all groups. Mean levels below 6\% were reached 3 month after surgery in groups I and II, 6 month after surgery in group III and 2 years after surgery in group IV (Figure 5).

\section{Discussion}

In this study, we evaluated short- and long-term effects on T2DM in patients after BPD-DS. In general, we saw encouraging results. This is of major importance since T2DM is one of the most important obesity-related comorbidities. Diabetes itself leads to a vast amount of diseases and complications of different organs.

In a group of 21 obese patients with insulin-dependent T2DM, we evaluated insulin usage and $\mathrm{HbA}_{1 \mathrm{c}}$ levels 6 and 12 months after BPD-DS. These patients had used insulin for a mean time of 6.5 years, and they had a mean usage of 98 units of insulin per day. Twelve months after the operation, they were all free of insulin with normalized $\mathrm{HbA}_{1 \mathrm{c}}$ levels and without any dietary restriction (Figure 2). Most other studies on BPD/BPD-DS include a substantial fraction of patients with T2DM who do not need insulin at the time point of operation but they report similar results, diabetes is resolved in the majority of cases after 1 year $[1,12]$. Remarkably, 15 patients were free of insuline at the time point of discharge, where a weight reduction is not yet achieved and, in the other patients, a significant reduction of insulin doses could be observed.

In a second collective of patients, the HOMA-IR index was measured to obtain more information about changes of insulin sensitivity in the early postoperative phase [12]. HOMA-IR is a product of serum insulin and blood glucose level after $12 \mathrm{~h}$ of fasting with values $>2$ providing evidence for insulin resistance and levels $>5$ in patients with T2DM [11]. We saw that after BPD-DS, the insulin sensitivity determined by the HOMA-IR index increased rapidly and usually nearly normalized within few days after surgery. The restoration of insulin sensitivity was independent on severity and duration of diabetes and also independent on BMI and the usage of oral antidiabetic medication.

Finally, to further evaluate whether the remission of T2DM depends on the duration and severity of diabetes, 74 patients were divided into 4 groups according to their 
need for insulin: we could see that even patients with a long history of insulin-dependent T2DM have a good chance for remission. However, in the group with a usage of insulin $>10$ years the chance of remission is significantly lower and a small percentage of patients will not be completely free of insulin - probably due to secondary beta-cell failure. Nevertheless, $\mathrm{HbA}_{1 \mathrm{c}}$ of these patients is significantly lower and less insulin is required which helps to control diabetesassociated complications and organ damages. As a summary of the data, it can be said that a substantial fraction of the patients is free of insulin and shows an improved insulin sensitivity early after the operation, another fraction gets free of insulin in a 12-month period after the operation and a small fraction of long-term insulin users will not get free of insulin but nevertheless shows an improved metabolic status (less insulin needed, normal $\mathrm{HbA}_{1 \mathrm{c}}$-levels).

The effects of gastrointestinal surgery on glucose metabolism are not understood in detail. The restrictive effect of bariatric surgery and the reduced caloric intake might lead to an improvement of glucose homeostasis and T2DM in the long run. This effect is equivalent to a diet and accompanied by weight loss and can be seen after restrictive operations like gastric banding [13]. A more pronounced effect can be observed after gastric bypass surgery and BPD and occurs significantly earlier, actually a few days after surgery, long before a loss of excess weight is achieved. Other mechanisms beside the "dietary" effect must exist [14]. In the early postoperative period, those mechanisms are independent of weight loss, later on they might be additive to the dietary-effect which can be seen after restrictive surgery. The "foregut hypothesis" states that the exclusion of the duodenum and proximal jejunum from the transient of nutrients are crucial for this effect, since it has also been seen in diabetic patients after subtotal gastrectomy due to ulcus or cancer [15-17]. The neuronal, hormonal, or chemical signalling pathways which influence insulin secretion and glucose homeostasis, and, thus, provide a link between "foregut" and beta cells are subject of various studies.

The question that consequently arises is the following: should BPD-DS be performed in non-obese patients with poorly controlled diabetes? It is not self-evident to expect that we will observe similar results in non-obese patients, because obesity itself might change the patients' metabolic status and mechanisms of regulation as well as levels and effects of hormones.

However, we have seen very similar results concerning usage of insulin and $\mathrm{HbA}_{1 c}$ in a population of 16 patients with T2DM and a BMI $<35 \mathrm{~kg} / \mathrm{m}^{2}$. The next important issue is that the weight-reductive operations, which have been developed, have an antidiabetic effect but they might not be the optimal antidiabetic operations in non-obese patients. Consequently, procedures may have to be optimized for nonobese patients with T2DM, resulting in novel antidiabetic procedures. It has to be determined, for example, if less gastric restriction-or no gastric restriction, like in case of the isolated duodenal switch-leads to similar anti-diabetic results. It has to be found out where to place the distal anastomosis to obtain optimal anti-diabetic results and minimal side effects like diarrhea or nutritional deficiencies.
As seen in bariatric surgery for morbid obesity, there will not probably be only a single "anti-diabetic" operation but several options, depending on the patients' comorbidities, weight, and, most likely, also duration and therapy of the patients' diabetes.

As a conclusion, we characterized the short- and medium-term effects of BPD-DS on T2DM. Preliminary results also show a good anti-diabetic effect in non-morbidly obese patients. Indications, mechanisms of action, and development of new surgical procedures to treat diabetes will be of major interest in the near future.

\section{References}

[1] H. Buchwald, Y. Avidor, E. Braunwald et al., "Bariatric surgery: a systematic review and meta-analysis," Journal of the American Medical Association, vol. 292, no. 14, pp. 1724-1737, 2004.

[2] F. Rubino, "Is type 2 diabetes an operable intestinal disease? A provocative yet reasonable hypothesis," Diabetes Care, vol. 31, pp. S290-S296, 2008.

[3] G. Noya, M. L. Cossu, M. Coppola et al., "Biliopancreatic diversion preserving the stomach and pylorus in the treatment of hypercholesterolemia and diabetes type II: results in the first 10 cases," Obesity Surgery, vol. 8, no. 1, pp. 67-72, 1998.

[4] R. Cohen, J. S. Pinheiro, J. L. Correa, and C. A. Schiavon, "Laparoscopic Roux-en-Y gastric bypass for BMI $<35 \mathrm{~kg} / \mathrm{m}^{2}$ : a tailored approach," Surgery for Obesity and Related Diseases, vol. 2, no. 3, pp. 401-404, 2006.

[5] R. V. Cohen, C. A. Schiavon, J. S. Pinheiro, J. L. Correa, and F. Rubino, "Duodenal-jejunal bypass for the treatment of type 2 diabetes in patients with body mass index of $22-34 \mathrm{~kg} / \mathrm{m}^{2}:$ a report of 2 cases," Surgery for Obesity and Related Diseases, vol. 3, no. 2, pp. 195-197, 2007.

[6] "Standards of medical care in diabetes-2009," Diabetes Care, vol. 32, supplement 1, pp. S13-S61, 2009.

[7] “Standards of medical care in diabetes-2010," Diabetes Care, vol. 33, supplement 1, pp. S11-S61, 2010.

[8] F. Rubino, L. M. Kaplan, P. R. Schauer, and D. E. Cummings, "The diabetes surgery summit consensus conference: recommendations for the evaluation and use of gastrointestinal surgery to treat type 2 diabetes mellitus," Annals of Surgery, vol. 251, no. 3, pp. 399-405, 2010.

[9] D. S. Hess, D. W. Hess, and R. S. Oakley, "The biliopancreatic diversion with the duodenal switch: results beyond 10 years," Obesity Surgery, vol. 15, no. 3, pp. 408-416, 2005.

[10] D. S. Hess and D. W. Hess, "Biliopancreatic diversion with a duodenal switch," Obesity Surgery, vol. 8, no. 3, pp. 267-282, 1998.

[11] D. R. Matthews, J. P. Hosker, A. S. Rudenski et al., "Homeostasis model assessment: insulin resistance and $\beta$-cell function from fasting plasma glucose and insulin concentrations in man," Diabetologia, vol. 28, no. 7, pp. 412-419, 1985.

[12] N. Scopinaro, G. M. Marinari, G. B. Camerini, F. S. Papadia, and G. F. Adami, "Specific effects of biliopancreatic diversion on the major components of metabolic syndrome: a long-term follow-up study," Diabetes Care, vol. 28, no. 10, pp. 2406-2411, 2005.

[13] J. B. Dixon, P. E. O’Brien, J. Playfair et al., "Adjustable gastric banding and conventional therapy for type 2 diabetes: a randomized controlled trial," Journal of the American Medical Association, vol. 299, no. 3, pp. 316-323, 2008. 
[14] W. J. Pories, M. S. Swanson, K. G. MacDonald et al., "Who would have thought it? An operation proves to be the most effective therapy for adult-onset diabetes mellitus," Annals of Surgery, vol. 222, no. 3, pp. 339-352, 1995.

[15] F. Rubino, A. Forgione, D. E. Cummings et al., "The mechanism of diabetes control after gastrointestinal bypass surgery reveals a role of the proximal small intestine in the pathophysiology of type 2 diabetes," Annals of Surgery, vol. 244, no. 5, pp. 741-749, 2006.

[16] M. N. Friedman, A. J. Sancetta, and G. J. Magovern, "The amelioration of diabetes mellitus following subtotal gastrectomy," Surgery, gynecology \& obstetrics, vol. 100, no. 2, pp. 201-204, 1955.

[17] L. Angervall, G. Dotevall, and H. Tillander, "Amelioration of diabetes mellitus following gastric resection," Acta medica Scandinavica, vol. 169, pp. 743-748, 1961. 


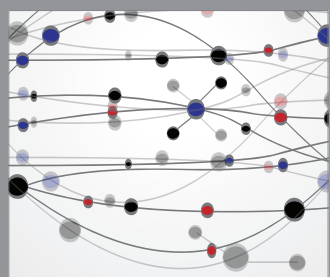

The Scientific World Journal
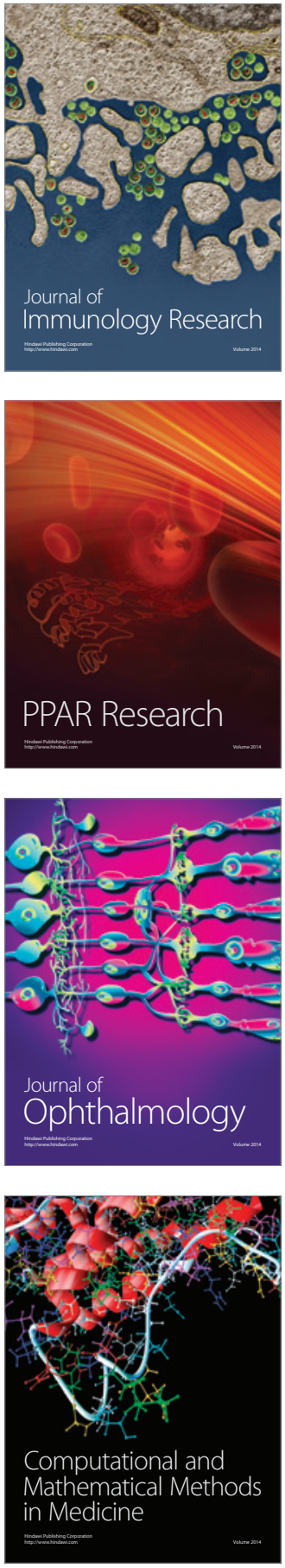

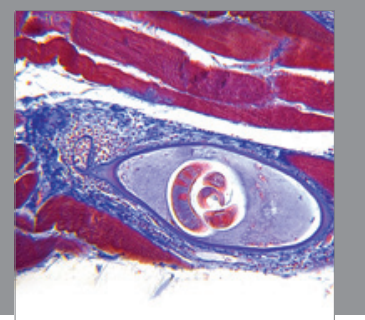

Gastroenterology

Research and Practice
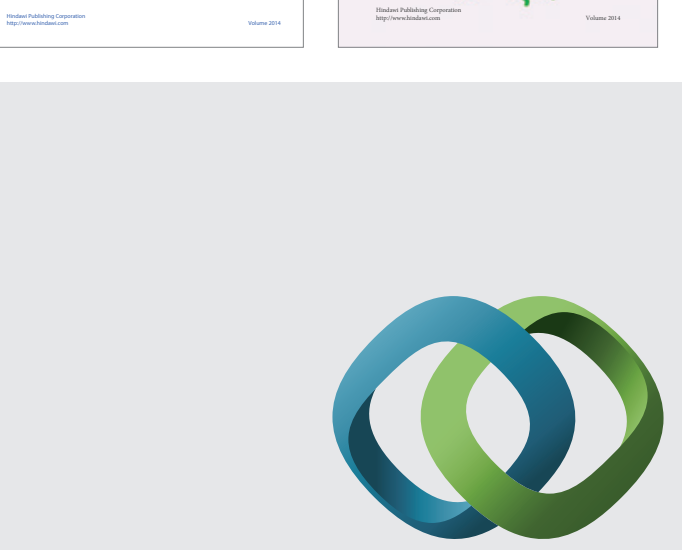

\section{Hindawi}

Submit your manuscripts at

http://www.hindawi.com
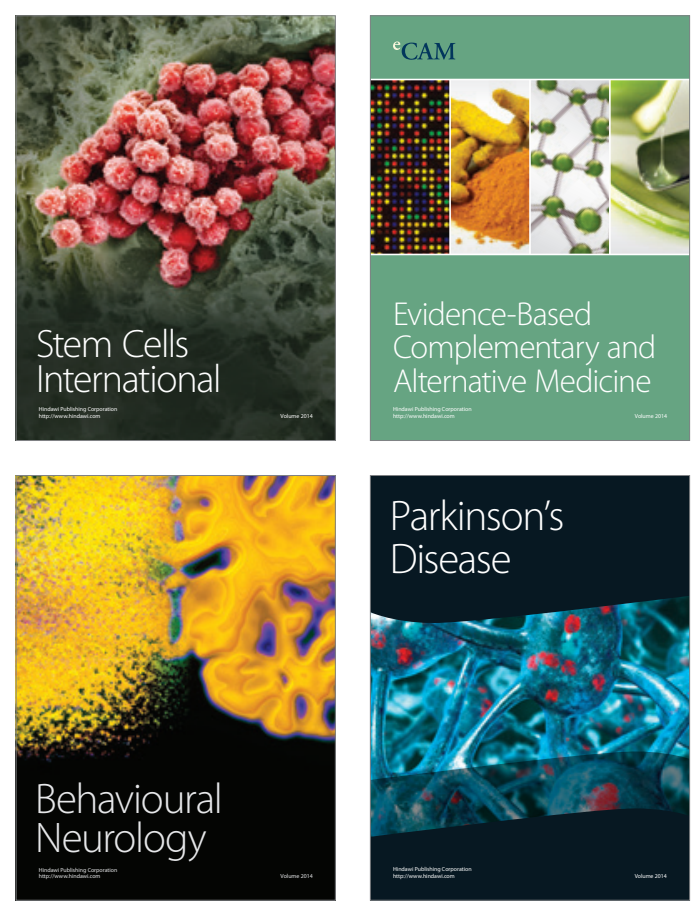

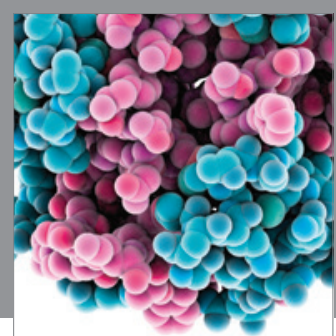

Journal of
Diabetes Research

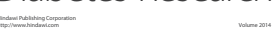

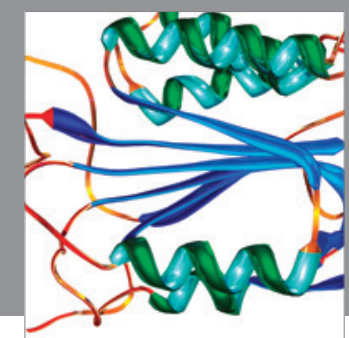

Disease Markers
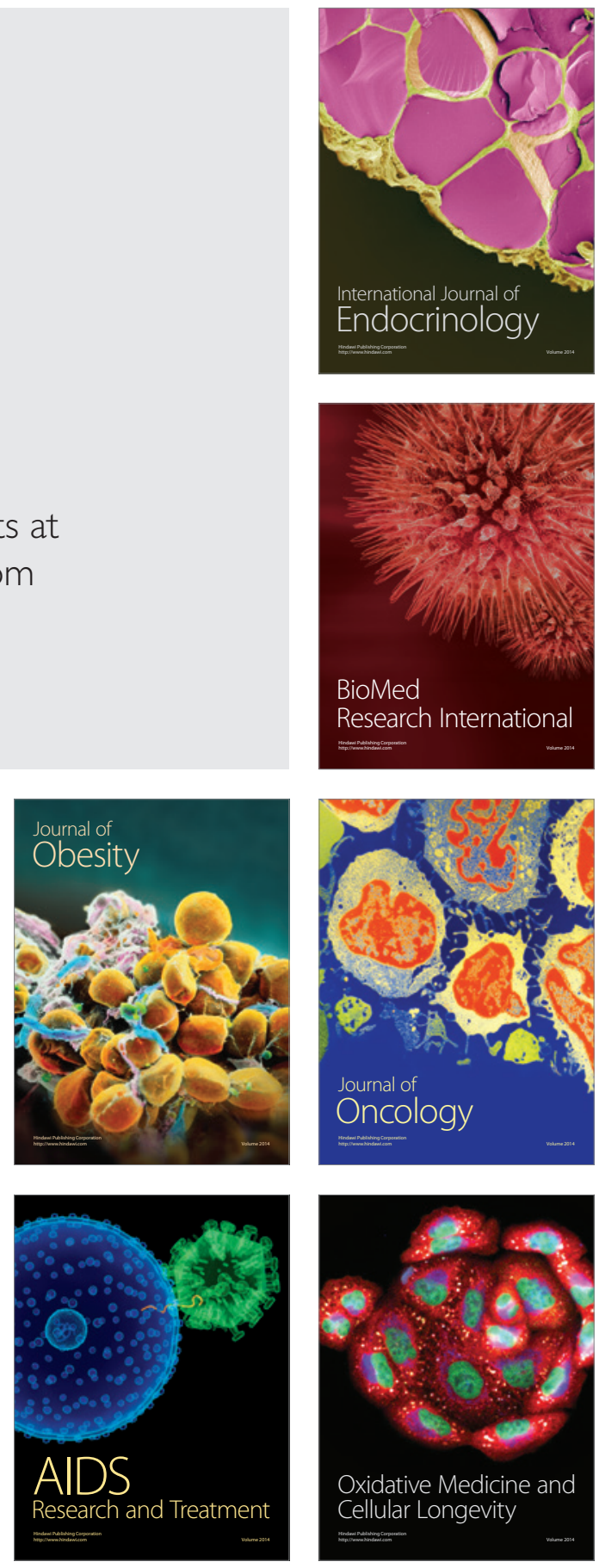